\title{
Nivel de conocimiento del idioma quechua, lengua indígena viva, en los estudiantes de la Facultad de Medicina de la Universidad Nacional Mayor de San Marcos \\ Level of knowledge of indigenous language, quechua, in the students at the Faculty of Medicine of the National University of San Marcos
}

\author{
Roberto Shimabuku Azato ${ }^{1}$, Martha Martina Chávez ${ }^{1}$, Ana Delgado Vásquez ${ }^{1}$, \\ Daniel Angulo Poblete ${ }^{1}$, Emma Salazar Salvatierra ${ }^{1}$ \\ Facultad de Medicina, Universidad Nacional Mayor de San Marcos. Lima, Perú.
}

An Fac med. 2018;79(3):264-6 / http://dx.doi.org/10.15381/anales.v79i3.15321

Correspondencia:

Roberto Shimabuku Azato

rshimabukua@unmsm.edu.pe

Recibido: 7 de agosto 2018

Aprobado: 25 de agosto 2018

Conflictos de interés: Los autores

declaran no tener conflictos de interés

Fuentes de financiamiento:

Autofinanciado

Citar como: Shimabuku R, Martina M, Delgado A, Angulo D, Salazar E. Nivel de conocimiento del idioma quechua, lengua indígena viva, en los estudiantes de la Facultad de Medicina de la Universidad Nacional Mayor de San Marcos. An Fac med. 2018;79(3):264-6. DOI: http://dx.doi.org/10.15381/anales. v79i3.15321

\section{Sr. Editor}

El quechua es la lengua originaria con más usuarios en el Perú, con alrededor de 4 millones de hablantes según el censo del año 2007. Coexiste con el idioma español en muchas regiones de la sierra, donde es el idioma predominante, y es usado cotidianamente por los pacientes o usuarios de los servicios de salud. En la Facultad de Medicina de la Universidad Nacional de San Antonio Abad del Cusco, que empezó a funcionar el año 1980, el quechua es un curso obligatorio. El $87,1 \%$ de los egresados de las primeras ocho promociones consideraron importante o muy importante conocer dicho idioma ${ }^{1}$. Asimismo, diversas publicaciones en nuestro medio, dan cuenta sobre la importancia de conocer idiomas nativos venciendo prejuicios $^{2,3,4}$.

Aunque hasta este momento no se ha establecido con certeza el nivel de conocimiento del idioma quechua entre nuestros estudiantes de ciencias de la salud, consideramos de importancia tener un punto de partida para iniciar la mejora en la capacidad de interactuar de nuestros futuros profesionales con los usuarios en la atención de la salud. Así, realizamos un estudio observacional, transversal, en la Facultad de Medicina de la Universidad Nacional Mayor de San Marcos que cuenta con cinco escuelas profesionales relacionadas a ciencias de la salud: medicina humana, obstetricia, enfermería, nutrición y tecnología médica. Se aplicó una encuesta anónima y voluntaria a todos los estudiantes ingresantes a las carreras de ciencias de la salud del año académico 2017, a quienes se explicó los objetivos del estudio y se obtuvo la respectiva autorización. La encuesta fue validada previamente por expertos en la docencia. El proyecto fue aprobado por el Comité de Ética de la Facultad de Medicina. La encuesta incluyó preguntas cerradas con opciones múltiples y una pregunta abierta acerca de la importancia del manejo del idioma quechua. Al final se obtuvieron respuestas que se analizaron según la naturaleza de las variables.

Los encuestados (442) representaron el $75 \%$ de los estudiantes ingresantes a la Facultad de Medicina en el año 2017. La edad promedio de los estudiantes fue de 19,10 $\pm 2,034$ años, con un rango de los 15 a 36 años de edad. El mayor porcentaje fue el grupo de 18 a 20 años (71\%). Hubo un predominio del sexo femenino $(65,4 \%)$. La mayoría procedía de la costa $(89,3 \%)$. En relación a la escuela profesional de procedencia, el $29 \%$ fueron de tecnología médica, el $28,5 \%$ de medicina, el $17 \%$ de obstetricia, el 14,9\% de enfermería y el 10,6\% de la escuela de nutrición.

Respecto a las características relacionadas al uso del quechua, en el 78,9\% (345) manifestó que los padres no hablan quechua; lo cual se eleva en el caso de las madres que llega a $80,3 \%$ (350). Un porcentaje importante, 42,9\% (187) de los estudiantes revelaron que viven con familias que hablan quechua. A las preguntas dirigidas si conocen o no el quechua, sólo un 10,1\% (37) de los estudiantes manifestó hablar, leer, o comprender quechua; y de 
éstos, el 76,5\% (28/37) sí lo usan. Según refirieron, el lugar donde mayormente usan el quechua fue dentro de casa, que llega a un 78,6\% (22/28). Debe destacarse que el $97,9 \%(421 / 433)$ de la población estudiada consideró importante el uso del quechua. El conocimiento de los ingresantes de la Facultad de Medicina (hablar, leer o comprender) se asoció significativamente con el hecho de tener un padre, madre o vivir con familia que habla quechua $(p<0,001)$; no así para el sexo y el lugar de procedencia (Tabla 1 ). Resulta interesante señalar que la importancia que le asignan los estudiantes al quechua es independiente del uso que tienen sobre el mismo.

En la base de datos oficial de pueblos indígenas $u$ originarios del Ministerio de Cultura se refiere que a principios del siglo XX el 60\% de la población peruana hablaba quechua y a principios del siglo XXI solo el $15 \%$ era quechua hablante, siempre coexistiendo con el castellano o español ${ }^{5}$. Estudios recientes reportaron que el idioma quechua se enseña en pocas escuelas de medicina: 3 de 25 escuelas en una investigación ${ }^{6}$, y 6 de 36 en otra ${ }^{7}$. Sólo en 4 escuelas era requisito obligatorio para titularse de médico cirujano. En la Facultad de Medicina de la Universidad Nacional Mayor de San Marcos, en las escuelas de enfermería, nutrición y obstetricia, el quechua existe como curso electivo, no obligatorio, con 2 o 3 créditos académicos.

En otros países, como Paraguay, país pequeño donde el guaraní es un idioma nativo, usado por cerca del 50\% de la población, de los estudiantes de medicina del hospital universitario de Paraguay sólo el 8,5\% comprendían y se expresaban correctamente en guaraní, 33\% no comprendían ni se expresaban en guaraní y 58,4\% comprendían o se expresaban parcialmente ${ }^{8}$. El español y el guaraní son consideramos idiomas oficiales en dicho país.

El peligro que supone la extinción de una lengua originaria como lo es el quechua -sobre la que se podría afirmar ha sido relegado por el Estado y la sociedad- constituye un arraigado problema ${ }^{9}$. Algunos progresos se dan periódicamente. La anterior Ley Universitaria 23733 de 1983 exigía como requisito para graduarse como Doctor dos idiomas extranjeros. La Ley Universitaria actual 30220 promulgada el año 2014 también exige dos idiomas extranjeros, pero una de ellas puede ser sustituida por una lengua nativa, normativa que ha sido recogida también en el actual Estatuto de la Universidad Nacional Mayor de San Marcos.

Nuestros estudiantes deberían tener la capacidad de aplicar los conocimientos a la práctica, y facilitar así la comunicación del futuro profesional de la salud con los usuarios de la atención de salud, sus familiares, o ambos, en el marco de los derechos de las personas a su salud y el buen trato, mejorando así la calidad de la atención. Usar el quechua como herramienta de comunicación con los pacientes debe facilitar la comprensión de los diagnósticos y tratamientos y difundir mejor los planes de prevención y promoción de la salud en la comunidad.

Tabla 1. Características de los estudiantes de la Facultad de Medicina de la Universidad Nacional Mayor de San Marcos en relación al conocimiento del quechua, 2017.

\begin{tabular}{|c|c|c|c|c|}
\hline \multirow{2}{*}{ Características } & \multicolumn{2}{|c|}{ Conocimiento quechua } & \multirow{2}{*}{ Total } & \multirow{2}{*}{ Valor $p^{*}$} \\
\hline & No habla, no lee, ni comprende & Habla, lee o comprende & & \\
\hline \multicolumn{5}{|l|}{ Sexo } \\
\hline Femenino & 196 & 21 & 217 & 0,757 \\
\hline Masculino & 134 & 16 & 150 & \\
\hline \multicolumn{5}{|c|}{ Lugar de nacimiento } \\
\hline Costa & 289 & 31 & 320 & 0,250 \\
\hline Sierra & 30 & 6 & 36 & \\
\hline Selva & 9 & 0 & 9 & \\
\hline \multicolumn{5}{|c|}{ Padre habla quechua } \\
\hline $\mathrm{Si}$ & 51 & 18 & 69 & $<0,001$ \\
\hline No & 274 & 19 & 293 & \\
\hline \multicolumn{5}{|c|}{ Madre habla quechua } \\
\hline $\mathrm{Si}$ & 43 & 25 & 68 & $<0,001$ \\
\hline No & 281 & 12 & 293 & \\
\hline \multicolumn{5}{|c|}{ Vive con familia que habla quechua } \\
\hline $\mathrm{Si}$ & 117 & 32 & 149 & $<0,001$ \\
\hline No & 207 & 5 & 212 & \\
\hline \multicolumn{5}{|c|}{ Si considera importante el quechua } \\
\hline $\mathrm{Si}$ & 309 & 37 & 346 & 0,300 \\
\hline No & 9 & 0 & 9 & \\
\hline
\end{tabular}

${ }^{*}$ Chi Cuadrado 


\section{REFERENCIAS BIBLIOGRÁFICAS}

1. Saco S, Villavicencio E. Situación actual y perspectivas de los médicos egresados de las primeras ocho promociones d la Facultad de Medicina de la Universidad Nacional de San Antonio Abad del Cusco (1989-1994). SITUA. 1999;7(14):1-15.

2. Oyola-Garcia AE, Quispe-llanzo M. La verdad es que no queremos aprender. An Fac med. 2016; 77(3): 293-94. DOI: http://dx.doi.org/10.15381/ anales.v77i3.12428

3. Alarco J. ¿Es necesaria la enseñanza de idioma quechua en nuestra facultad? (Editorial). Rev méd Panacea. 2014; 4(2): 29-30.
4. Garcia Nuñez O. Simita Yachakuy. Revista Somos, El Comercio (Lima). 18 de febrero 2017; pp. 32-36

5. Ministerio de Cultura del Perú [Internet]. Pueblos Quechuas. Base de Datos Oficial de Pueblos Indigenas u Originarios. Lima; 2018 [Fecha de acceso 24 de julio de 2018]. Disponible en: http:// bdpi.cultura.gob.pe/pueblo/quechuas

6. Santos-Revilla G. Enseñanza de lenguas originarias en las facultades de medicina peruanas [carta]. Rev Peru Med Exp Salud Publica. 2016;33(1):1834. DOI: 10.17843/rpmesp.2016.331.2021.

7. Arce-Villalobos LR, Toro-Huamanchumo CJ, Melgarejo-Castillo A, Taype-Rondan A. Enseñanza de idiomas en escuelas de medicina humana de
Perú. Rev. Fac. Med. 2017;65(4):583-8. DOI: http:// dx.doi.org/10.15446/revfacmed.v65n4.59416.

8. Jiménez HJ, Delgadillo $L$, Campuzano de Rolón A, Jiménez D, de Samudio A, Agüero A, Radice C, Jiménez-Britez G. Knowledge of the guarani language at a university hospital in Paraguay. Medwave. 2018;18(2):e7200. DOI: 10.5867/medwave.2018.02.7200

9. Blácido R [Internet]. La situación del quechua en el Perú y su inclusión en el sistema educativo. Repositorio Institucional Universidad de Lima. Lima; 2016. [Fecha de acceso 24 de julio de 2018]. Disponible en: http://repositorio.ulima.edu.pe/ handle/ulima/4743 